\title{
THE EFFECT OF THE RANDOM MAGNETIC FIELD COMPONENT ON THE PARKER INSTABILITY
}

\author{
JONGSOO KIM \\ Korea Astronomy Observatory, 61-1, Hwaam-Dong, Yusong-Ku, Taejon 305-348, Korea; and NCSA, University of Illinois \\ at Urbana-Champaign, 405 North Mathews Avenue, Urbana, IL 61801; jskim@ncsa.uiuc.edu \\ AND \\ DONGSU RYU \\ Department of Astronomy and Space Science, Chungnam National University, 220 Kung-Dong, \\ Yusong-Ku, Daejeon 705-764, Korea; ryu@ canopus.chungnam.ac.kr \\ Received 2001 July 2; accepted 2001 September 18; published 2001 October 10
}

\begin{abstract}
The Parker instability is considered to play important roles in the evolution of the interstellar medium. Most studies on the development of the instability so far have been based on an initial equilibrium system with a uniform magnetic field. However, the Galactic magnetic field possesses a random component in addition to the mean uniform component, with comparable strength of the two components. Parker and Jokipii have recently suggested that the random component can suppress the growth of small wavelength perturbations. Here we extend their analysis by including gas pressure, which was ignored in their work, and study the stabilizing effect of the random component in the interstellar gas with finite pressure. Following Parker and Jokipii, we model the magnetic field as a mean azimuthal component, $B(z)$, plus a random radial component, $\epsilon(z) B(z)$, where $\epsilon(z)$ is a random function of height from the equatorial plane. We show that for the observationally suggested values of $\left\langle\epsilon^{2}\right\rangle^{1 / 2}$, the tension due to the random component becomes important, so that the growth of the instability is either significantly reduced or completely suppressed. When the instability still works, the radial wavenumber of the most unstable mode is found to be zero. That is, the instability is reduced to be effectively two-dimensional. We discuss briefly the implications of our finding.
\end{abstract}

Subject headings: instabilities — ISM: clouds — ISM: magnetic fields — MHD

\section{INTRODUCTION}

The magnetostatic equilibrium of the system of the interstellar gas and magnetic field under the vertical gravitational field of the Galaxy has been shown to be unstable (Parker 1966, 1967). The physical mechanism for the instability relies on the fact that a light fluid (represented by the magnetic field) supports a heavy fluid (represented by the gas) and the configuration tends to overturn. It has similarities to the RayleighTaylor instability, when a true light fluid supports a heavy fluid. In the Rayleigh-Taylor instability, the fastest growing mode has an infinite perturbation wavenumber. However, taking into account a "unidirectional" magnetic field along the azimuthal direction in the Galactic disk, Parker (1966) showed that the magnetic tension stabilizes large wavenumber perturbations and results in a preferred, finite wavenumber. But when the perturbations along the radial direction are allowed, those with an infinite radial wavenumber prevail (Parker 1967). As a result, the structures formed by the Parker instability are expected to be elongated, and Kim et al. (1998) confirmed it through three-dimensional simulations for the nonlinear evolution of the Parker instability.

The Parker instability in the interstellar medium (ISM) has been thought to be a viable mechanism in forming giant molecular clouds (GMCs) in the Galaxy (see, e.g., Appenzeller 1974; Mouschovias, Shu, \& Woodward 1974; Blitz \& Shu 1980). However, the work of Kim et al. (1998) raised negative points on that. In addition to the fact that sheetlike structures with the smallest scale in the radial direction are formed, they found that the enhancement factor of column density is at most $\sim 2$. The second density issue is eased by noting that the interstellar gas can be further susceptible to the thermal instability, as pointed out in Parker \& Jopikii (2000), followed by the gravitational instability. However, the first structural issue, which is the direct result of the infinitesimal radial wavenumber, does not easily go away.

Several ideas on effects that could suppress the maximally unstable nature of the mode with an infinite wavenumber have been suggested. One of them is to invoke a "stochastic magnetic field" (Parker \& Jopikii 2000), which represents the random component of the Galactic magnetic field. Using a field composed of the usual mean component and a transverse component whose strength is weak and random, they showed that in "cold plasma" (without gas pressure) the weak, random component exerts a significant stabilizing effect on the perturbations with small transverse wavelengths. The physical mechanism is the following. Although weak, the tension of the transverse component that is incurred by the vertical gas motions is strong enough to reinstate the gas. They suggested the possibility of preferred modes with finite transverse (radial) wavenumbers. Such modes would result in broadened structures, which would resemble more the morphology of the GMCs. Their stochastic field model is promising in the sense that (1) it is consistent with the turbulent picture of the ISM (see, e.g., Minter \& Spangler 1996) and (2) it is supported by the observations of the magnetic field in our Galaxy and spiral galaxies (see, e.g., Beck et al. 1996; Zweibel \& Heiles 1997). However, their cold plasma approximation needs to be improved.

The purpose of this Letter is to analyze fully the effects of the random component of the magnetic field on the Parker instability in a medium with finite gas pressure. We find the rather surprising results that the random component either reduces the growth of the instability significantly or suppresses it completely. And the most unstable mode has a vanishing radial wavenumber. The plan of the Letter is as follows: linear stability analysis is carried out by analyzing the dispersion relation in $\S 2$, and a summary and discussion follow in $\S 3$. 


\section{LINEAR STABILITY ANALYSIS}

We consider the stability of an equilibrium system where gas is supported by its own and magnetic pressures against a "uniform" gravity, $g$, in the negative $z$ (vertical) direction. With realistic gravities, different growth rates and wavelengths of unstable modes would result (see, e.g., Kim, Hong, \& Ryu 1997), but they make the analysis much more involved. In addition, we expect the qualitative features of the stability would not be affected by details of gravity. For the magnetic field configuration, the stochastic model suggested by Parker \& Jopikii (2000) is adopted. It is composed of a mean component, $B(z)$, in the $y$ (azimuthal) direction and a random component, $\epsilon(z) B(z)$, in the $x$ (radial) direction. The quantity $\epsilon(z)$ is a random function of $z$ with zero mean. One assumption made on $\epsilon(z)$ is that the correlation length is small compared to the vertical scale height of the system. So in the equations below, the local average is taken by integrating over $z$ for a vertical scale greater than the correlation length of $\epsilon(z)$ but smaller than the scale height. Then, the dispersion $\left\langle\epsilon^{2}\right\rangle$ is taken as a constant, which becomes a free parameter of the analysis. With finite gas pressure, $p$, the magnetohydrostatic equilibrium is governed by

$$
\frac{d}{d z}\left[p+\left(1+\left\langle\epsilon^{2}\right\rangle\right) \frac{B^{2}}{8 \pi}\right]=-\rho g
$$

where $\rho$ is gas density. Two further assumptions are made, which are usual in the analysis of the Parker instability: (1) an isothermal equation of state, $p=a_{s}^{2} \rho$, where $a_{s}$ is the isothermal speed, and (2) a constant ratio of magnetic to gas pressures, $\alpha=\left(1+\left\langle\epsilon^{2}\right\rangle\right) B^{2} / 8 \pi p$. Then exponential distributions of density, gas pressure, and magnetic pressure are obtained:

$$
\frac{\rho(z)}{\rho(0)}=\frac{p(z)}{p(0)}=\frac{B^{2}(z)}{B^{2}(0)}=\exp \left(-\frac{|z|}{H}\right),
$$

where the $e$-folding scale height, $H$, is given by $(1+\alpha) a_{s}^{2} / g$.

The above equilibrium state is disturbed with an infinitesimal perturbation. The perturbed system is assumed to be isothermal too. Since linearized perturbation equations for the case without gas pressure were already derived (Parker \& Jopikii 2000), the detailed derivation is not repeated here. Instead, a reduced form in terms of velocity perturbations, $\left(v_{x}, v_{y}, v_{z}\right)$, is written as follows:

$$
\begin{aligned}
\frac{\partial^{2} v_{x}}{\partial t^{2}}= & a_{s}^{2} \frac{\partial}{\partial x}\left(\frac{\partial v_{x}}{\partial x}+\frac{\partial v_{y}}{\partial y}+\frac{\partial v_{z}}{\partial z}-\frac{v_{z}}{H}\right) \\
& +v_{\mathrm{A}}^{2}\left[\frac{\partial^{2} v_{x}}{\partial y^{2}}+\frac{\partial^{2} v_{x}}{\partial x^{2}}+\frac{\partial}{\partial x}\left(\frac{\partial v_{z}}{\partial z}-\frac{v_{z}}{2 H}\right)-\frac{\left\langle\epsilon^{2}\right\rangle}{2 H} \frac{\partial v_{z}}{\partial x}\right], \\
\frac{\partial^{2} v_{y}}{\partial t^{2}}= & a_{s}^{2} \frac{\partial}{\partial y}\left(\frac{\partial v_{x}}{\partial x}+\frac{\partial v_{y}}{\partial y}+\frac{\partial v_{z}}{\partial z}-\frac{v_{z}}{H}\right)+v_{\mathrm{A}}^{2}\left\{-\frac{1}{2 H} \frac{\partial v_{z}}{\partial y}\right. \\
& \left.+\left\langle\epsilon^{2}\right\rangle\left[\frac{\partial^{2} v_{y}}{\partial x^{2}}+\frac{\partial^{2} v_{y}}{\partial y^{2}}+\frac{\partial}{\partial y}\left(\frac{\partial v_{z}}{\partial z}-\frac{v_{z}}{2 H}\right)\right]\right\},
\end{aligned}
$$

and

$$
\begin{aligned}
\frac{\partial^{2} v_{z}}{\partial t^{2}}= & a_{s}^{2} \frac{\partial}{\partial z}\left(\frac{\partial v_{x}}{\partial x}+\frac{\partial v_{y}}{\partial y}+\frac{\partial v_{z}}{\partial z}-\frac{v_{z}}{H}\right) \\
& +v_{\mathrm{A}}^{2}\left\{\frac{\partial^{2} v_{z}}{\partial y^{2}}+\left(\frac{\partial}{\partial z}-\frac{1}{H}\right)\left[\left(\frac{\partial}{\partial z}-\frac{1}{2 H}\right) v_{z}+\frac{\partial v_{x}}{\partial x}\right]\right. \\
& +\frac{1}{2 H}\left(\frac{\partial v_{x}}{\partial x}+\frac{\partial v_{y}}{\partial y}+\frac{\partial v_{z}}{\partial z}-\frac{v_{z}}{H}\right)+\left\langle\epsilon^{2}\right\rangle \\
& \left.\times\left[\left(\frac{\partial}{\partial z}-\frac{1}{H}\right)\left(\frac{\partial}{\partial z}-\frac{1}{2 H}\right) v_{z}+\left(\frac{\partial}{\partial z}-\frac{1}{H}\right) \frac{\partial v_{y}}{\partial y}+\frac{\partial^{2} v_{z}}{\partial x^{2}}\right]\right\} .
\end{aligned}
$$

Here $v_{\mathrm{A}}$ is the Alfvén speed, $B / \sqrt{4 \pi \rho}$, which is constant over $z$. Note that the linearized perturbation equations for the cold plasma (eqs. [12]-[14] in Parker \& Jopikii 2000) are recovered from the above equations by (1) dropping out the terms with $a_{s}$ and (2) noting that the scale height of magnetic field ( $\Lambda$ of their notation) is twice larger than that of gas ( $H$ of our notation).

The normal mode solution takes the following form:

$$
\begin{aligned}
\left(v_{x}, v_{y}, v_{z}\right)= & \left(D_{x}, D_{y}, D_{z}\right) \\
& \times \exp \left(\frac{t}{\tau}+i k_{x} x+i k_{y} y+i k_{z} z+\frac{z}{2 H}\right),
\end{aligned}
$$

where $D_{x}, D_{y}$, and $D_{z}$ are constants. Taking $H$ and $H / a_{s}$ as the normalization units of length and time, respectively, the dimensionless growth rate, $\Omega=H /\left(a_{s} \tau\right)$, and the dimensionless wavenumber, $\left(q_{x}, q_{y}, q_{z}\right)=H\left(k_{x}, k_{y}, k_{z}\right)$, are defined. Substituting equation (6) into equations (3)-(5) and imposing the condition of a nontrivial solution, we get the dispersion relation

$$
\Omega^{6}+C_{4} \Omega^{4}+C_{2} \Omega^{2}+C_{0}=0
$$

where the coefficients $C_{4}, C_{2}$, and $C_{0}$ are given by

$$
\begin{aligned}
C_{4}= & 2 \alpha q_{y}^{2}+(2 \alpha+1)\left(q_{x}^{2}+q_{y}^{2}+q_{z}^{2}+1 / 4\right) \\
& +2 \alpha\left\langle\epsilon^{2}\right\rangle\left(2 q_{x}^{2}+q_{y}^{2}+q_{z}^{2}+i q_{z} / 2\right),
\end{aligned}
$$

$$
\begin{aligned}
C_{2}= & \alpha(\alpha+1)\left[q_{x}^{2}+4 q_{y}^{2}\left(q_{x}^{2}+q_{y}^{2}+q_{z}^{2}\right)\right]+\alpha\left\langle\epsilon^{2}\right\rangle \\
& \times\left\{\alpha q_{x}^{2}+(2 \alpha+1) q_{y}^{2}+4 q_{x}^{2}\left[(2 \alpha+1)\left(q_{x}^{2}+q_{z}^{2}\right)\right.\right. \\
& \left.\left.+(4 \alpha+1) q_{y}^{2}\right]+4 \alpha q_{y}^{2}\left[2\left(q_{y}^{2}+q_{z}^{2}\right)+i q_{z} / 2\right]\right\} \\
& +4 \alpha^{2}\left\langle\epsilon^{2}\right\rangle^{2} q_{x}^{2}\left(q_{x}^{2}+q_{y}^{2}+q_{z}^{2}+i q_{z} / 2\right),
\end{aligned}
$$

and

$$
\begin{aligned}
C_{0}= & 2 \alpha^{2} q_{y}^{2}\left[2 q_{y}^{2}\left(q_{x}^{2}+q_{y}^{2}+q_{z}^{2}+1 / 4\right)-(\alpha+1)\left(q_{x}^{2}+q_{y}^{2}\right)\right] \\
& +2 \alpha^{2}\left\langle\epsilon^{2}\right\rangle\left\{\left[(\alpha+1) q_{x}^{4}+3(\alpha+1) q_{x}^{2} q_{y}^{2}+(2 \alpha+1) q_{y}^{4}\right]\right. \\
& \left.+4 q_{y}^{2}\left(q_{x}^{2}+q_{y}^{2}+q_{z}^{2}\right)\left[q_{x}^{2}+\alpha\left(q_{x}^{2}+q_{y}^{2}\right)\right]\right\}-\alpha^{2}\left\langle\epsilon^{2}\right\rangle^{2} q_{x}^{2} \\
& \times\left\{q_{x}^{2}+2 q_{y}^{2}-4\left(q_{x}^{2}+q_{y}^{2}+q_{z}^{2}\right)\left[q_{x}^{2}+2 \alpha\left(q_{x}^{2}+q_{y}^{2}\right)\right]\right\} .
\end{aligned}
$$


Equation (7) is a cubic equation of $\Omega^{2}$ with complex coefficients. For the case with vanishing vertical wavenumber $\left(q_{z}=0\right)$, all the $C$ coefficients become real, and the dispersion relation can be easily solved. For small vertical wavenumbers, the imaginary terms, $i\left\langle\epsilon^{2}\right\rangle q_{z}$ and $i\left\langle\epsilon^{2}\right\rangle^{2} q_{z}$ in $C_{4}$ and $C_{2}$, can be still ignored. This trick does not affect the marginal condition of the stability $(\Omega=0)$, since $C_{0}$ does not contain any imaginary term. Here we remind readers of the definition of $\alpha$. It is reserved in this Letter for the ratio of magnetic to gas pressures, whereas it was used for the dispersion of $\epsilon$ in Parker \& Jopikii (2000). For the dispersion, $\left\langle\epsilon^{2}\right\rangle$ is used in this Letter.

Two limiting cases can be considered, which enable us to check the validity of the above relation. The formula with $\left\langle\epsilon^{2}\right\rangle=0$ reduces to the dispersion relation for the original Parker instability (see, e.g., Parker 1967; Shu 1974). The values of $C$ without the terms containing $\left\langle\epsilon^{2}\right\rangle$ match exactly with the coefficients of equation (53) in Shu (1974), after imposing the isothermal condition $\gamma=1$. The other limiting formula is for the cold plasma with $p=0$ (Parker \& Jopikii 2000). As shown above, our linearized perturbation equations recover those for the cold plasma.

The full stability property can be analyzed by solving the above dispersion relation numerically. Figure 1 shows the stability diagram for $\alpha=1$. Equi- $\Omega^{2}$ contours with positive values corresponding to unstable modes are plotted on the $\left(q_{x}^{2}, q_{y}^{2}\right)$ plane for a few different values of $\left\langle\epsilon^{2}\right\rangle^{1 / 2} ; q_{z}=0$ has been set. Finite $q_{z}$ reduce the growth rate (see, e.g., Parker 1966). Three interesting points can be made: with increasing strength of the random component, (1) the domain of the instability in the $\left(q_{x}^{2}, q_{y}^{2}\right)$-plane shrinks, (2) the maximum growth rate decreases, and (3) the $q_{x \text {, max }}$, which gives the maximum growth rate, decreases and reduces to zero eventually. Note that without the random component, $\left\langle\epsilon^{2}\right\rangle^{1 / 2}=0$, the most unstable mode has the growth rate $\Omega^{2}=0.172$, and the radial wavenumber $q_{x, \max } \rightarrow \infty$.

The above points can be seen more clearly in Figure 2, which shows the growth rate and two horizontal wavenumbers of the most unstable modes as a function of $\left\langle\epsilon^{2}\right\rangle^{1 / 2}$ for three different values of $\alpha$. Again, $q_{z}=0$ has been set. Note that the scale height $H$ changes with $\alpha$. Hence, both the growth rate and wavenumber in real units scale as $1 /(1+\alpha)$. Even after this factor is taken into account, the maximum growth rate increases with $\alpha$, due to enhanced magnetic buoyancy. Two additional points can be made: (1) the critical value $\left\langle\epsilon^{2}\right\rangle_{c}^{1 / 2}$, above which the Parker instability disappears completely, is independent of $\alpha$, and it is computed as $1 / \sqrt{2}=0.707$ from the dispersion relation, and (2) the value of $\left\langle\epsilon^{2}\right\rangle^{1 / 2}$, above which the radial wavenumber of the most unstable mode vanishes, decreases with increasing $\alpha$. These are the consequences of different roles of uniform and random magnetic fields.

On the issue of the formation of GMCs, the most interesting range of the values would be $\left\langle\epsilon^{2}\right\rangle^{1 / 2} \sim 0.1$, which would result in $q_{x} \sim q_{y}$ for the most unstable modes (although it would depend on $\alpha$; see Fig. 2). Then, the structures formed as the result of the instability would be round, mimicking GMCs. However, observations suggest a larger random component. It is generally quoted that in the Galactic plane $0.5 \lesssim\left\langle\epsilon^{2}\right\rangle^{1 / 2} \lesssim 1$, with the strength of the total magnetic field $B \sim 3-4 \mu \mathrm{G}$ (see, e.g., Beck et al. 1996; Zweibel \& Heiles 1997). But others, such as Minter \& Spangler (1996), suggest somewhat smaller values such as $\left\langle\epsilon^{2}\right\rangle^{1 / 2} \sim \frac{1}{4}-\frac{1}{3}$. If $0.5 \lesssim\left\langle\epsilon^{2}\right\rangle^{1 / 2} \lesssim 1$, the instability disappears completely or the growth rate reduces significantly by more than $80 \%$. If $\left\langle\epsilon^{2}\right\rangle^{1 / 2} \sim \frac{1}{4}-\frac{1}{3}$, the growth rate reduces by

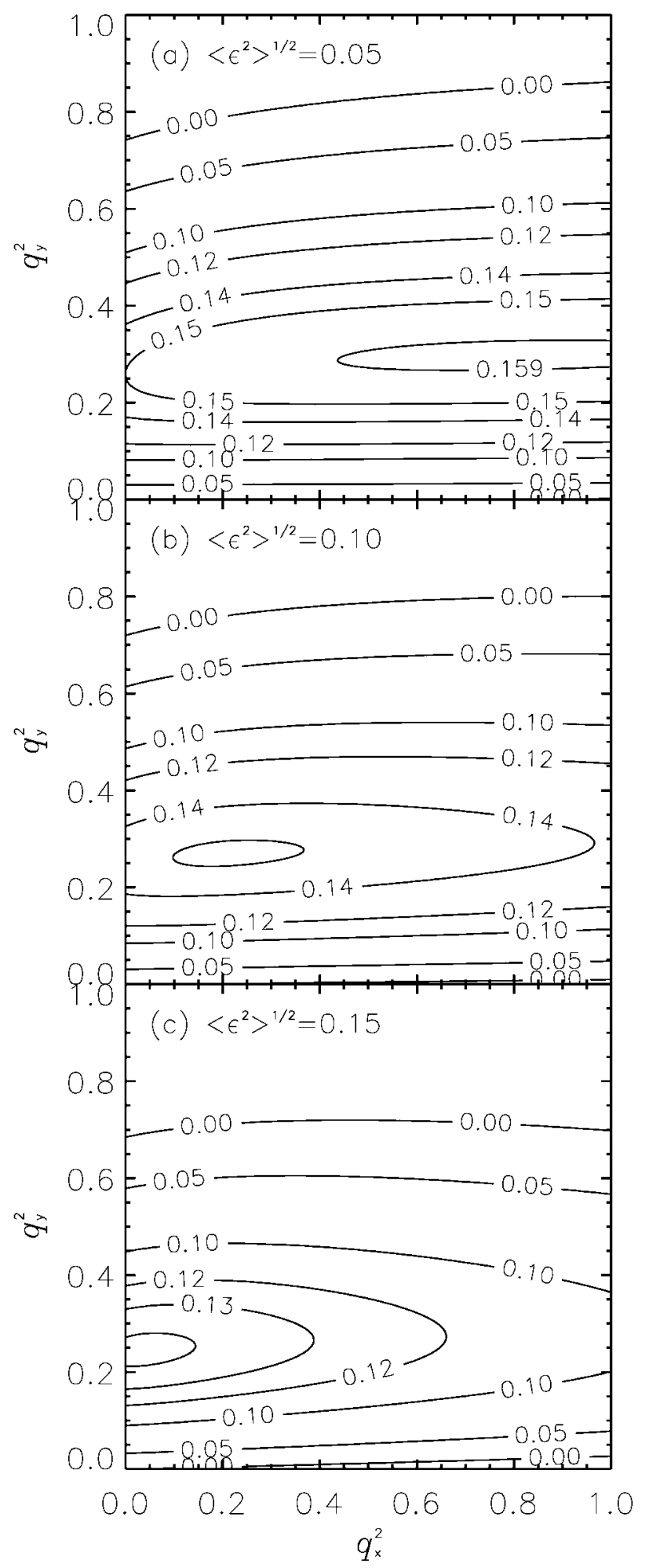

FIG. 1.-Equi- $\Omega^{2}$ contours on the $\left(q_{x}^{2}, q_{y}^{2}\right)$-plane for $(a)\left\langle\epsilon^{2}\right\rangle^{1 / 2}=0.05$, (b) $\left\langle\epsilon^{2}\right\rangle^{1 / 2}=0.10$, and $(c)\left\langle\epsilon^{2}\right\rangle^{1 / 2}=0.15$. In all plots, $\alpha=1$ and $q_{z}=0$ are used.

$30 \%-60 \%$. But in any case, the radial wavenumber of the most unstable mode shrinks to zero $\left(q_{x}=0\right)$. That is, the instability basically becomes two-dimensional in the plane defined by the azimuthal and vertical directions. This result is opposite to that of $\left\langle\epsilon^{2}\right\rangle^{1 / 2}=0$, where the dominant mode of the instability has vanishing radial wavelength $\left(q_{x} \rightarrow \infty\right)$. 


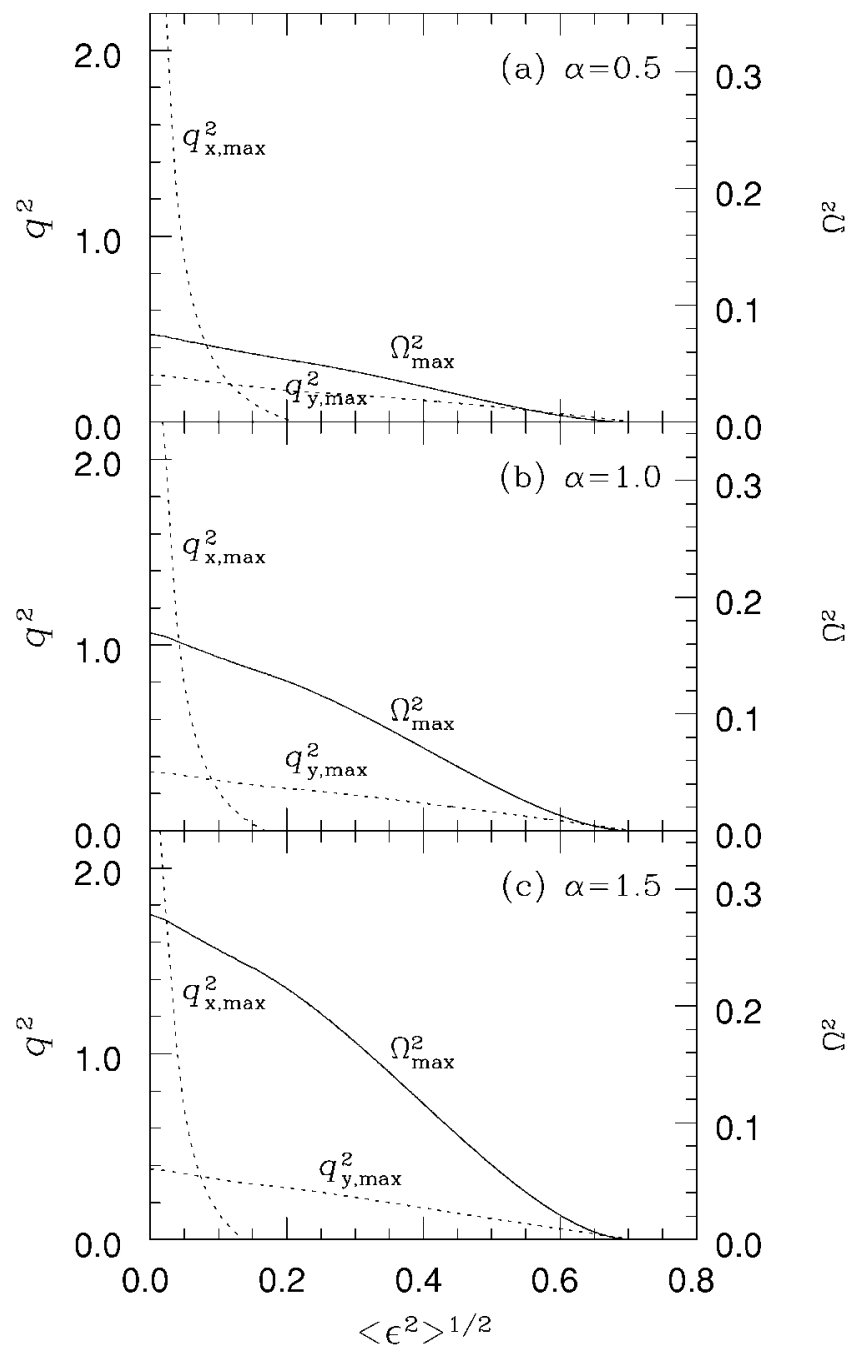

FIG. 2.-Maximum growth rate, $\Omega_{\max }^{2}$, and its two horizontal wavenumbers, $q_{x, \max }^{2}$ and $q_{y, \max }^{2}$, as a function of $\left\langle\epsilon^{2}\right\rangle^{1 / 2}$ for $(a) \alpha=0.5,(b) \alpha=1.0$, and (c) $\alpha=1.5 ; q_{z}=0$ for all the cases. Note that the growth rate and wavenumber in real units scale as $1 /(1+\alpha)$.

\section{DISCUSSION AND SUMMARY}

The Parker instability is induced by the magnetic buoyancy of the uniform component of magnetic field, while gas pressure and the random component exert stabilizing effects. The role of gas pressure is mainly exercising pressure force along the uniform component and setting finite wavenumbers for the instability along the uniform component direction. On the other hand, the random component threads rising and sinking slices across the uniform field. Its tension becomes stronger at larger wavenumbers. So the role of the random field is to suppress the growth of perturbations with large wavenumbers perpendicular to the uniform field.

Through the linear stability analysis, which includes both gas pressure and a random magnetic field, we have found that with the observationally favored values for the strength of the random component, $0.5 \lesssim\left\langle\epsilon^{2}\right\rangle^{1 / 2} \lesssim 1$, the tension of the random component that is incurred by the vertical gas motions is strong enough that the growth of the instability is either significantly reduced or completely suppressed. For smaller values, $\left\langle\epsilon^{2}\right\rangle^{1 / 2} \sim \frac{1}{4}-\frac{1}{3}$, which are suggested by others, the Parker instability is still operating but with reduced growth rate and vanishing radial wavenumber. With $\left\langle\epsilon^{2}\right\rangle^{1 / 2} \sim \frac{1}{4}-\frac{1}{3}$, by taking $H=160 \mathrm{pc}$ and $a_{s}=6.4 \mathrm{~km} \mathrm{~s}^{-1}$ (Falgarone \& Lequeux 1973), the growth timescale and the azimuthal wavelength of the most unstable mode are 70-95 Myr and $\sim 2.2 \mathrm{kpc}$, respectively. They are too large for the Parker instability to be a plausible mechanism for the formation of GMCs. But it is known that realistic gravity would reduce both (see, e.g., Kim et al. 1997). The more serious obstacle in the context of the GMC formation is the fact that the radial wavelength of the most unstable mode is infinity. This indicates the structures formed would be elongated, in this case, along the radial direction. But it is not clear whether such elongated structures would persist in the stage of the nonlinear development of the instability. That should be tested by numerical simulations.

The work was supported in part by KRF through grant KRF2000-015-DS0046. We thank R. Jokipii for discussions and $\mathrm{T}$. W. Jones for comments on the manuscript.

\section{REFERENCES}

Appenzeller, I. 1974, A\&A, 36, 99

Beck, R., Brandenburg, A., Moss, D., Shukurov, A., \& Sokoloff, D. 1996, ARA\&A, 34, 155

Blitz, L., \& Shu, F. H. 1980, ApJ, 238, 148

Falgarone, E., \& Lequeux, J. 1973, A\&A, 25, 253

Kim, J., Hong, S. S., \& Ryu, D. 1997, ApJ, 485, 228

Kim, J., Hong, S. S., Ryu, D., \& Jones, T. W. 1998, ApJ, 506, L139
Minter, A. H., \& Spangler, S. R. 1996, ApJ, 458, 194

Mouschovias, T. Ch., Shu, F. H., \& Woodward, P. R. 1974, A\&A, 33, 73

Parker, E. N. 1966, ApJ, 145, 811 1967, ApJ, 149, 535

Parker, E. N., \& Jokipii, J. R. 2000, ApJ, 536, 331

Shu, F. H. 1974, A\&A, 33, 55

Zweibel, E. G., \& Heiles, C. 1997, Nature, 385, 131 